\title{
Cover illustration: Eclipse ... when the sun turns to ashes
}

The ticking is loud, regular, relentless. People shift nervously, fussing with their telescopes, making small talk. Someone has a short wave radio tuned to a station in Boulder, Colorado, that broadcasts signals, providing the exact time, precise to the second. As the time grows closer, the small talk ceases. Finally, someone shouts, "First contact." All in attendance know that the disc of the moon has begun to slide across the face of the sun and will ultimately blot out the light of the sun as the celestial dance leads to a total solar eclipse.

After first contact, no change is obvious and excitement builds slowly since it takes about an hour and a half for the moon to completely occlude the sun to give totality. In the meantime there is plenty to do with telescopes and cameras. As darkness begins to surround us, when only a quarter of the sun remains, planets begin to shine through the dim light. Someone shouts "There's Mercury," difficult to see anytime because it is so close to the sun and lost in its light, or if visible at night, usually only shortly after sunset, and then only briefly to be seen near the horizon.

As second contact approaches, this time meaning totality, the buzz and whirl of equipment and the chatter between fellow observers grows into a crescendo. Someone shouts "Diamond ring," as the last light of the sun shines brightly like a diamond solitaire on a ring. It is only a heartbeat until totality. Shadow bands from the ripples in the earth's atmosphere scatter across the ground beneath us. Shouts of "Chromosphere!" and "Corona!" are accompanied by gasps and screams of awe as the magnificence of a total solar eclipse plunges its viewers into darkness and delight.

At totality the corona is no brighter than a full moon, and darkness has descended with all the attendant phenomena such as birds going to roost and stars twinkling. The feelings of awe, of humility, of joy take over.

All plans for photography, especially among the uninitiated, seem to be forgotten as the overwhelming spectacle of the earth's greatest natural event passes its spell over the group. Usually, eclipses last 1-3 minutes, with the umbra moving at about 3000 kilometres an hour along its predestined path. The longest eclipses may be well over 6 minutes. The time flies by as centreline passes overhead much like a silent and majestic cyclone. Then, with shouts of "Ten seconds to diamond ring" everyone prepares for the end of totality. Solar filters and appropriate glasses are prepared since ocular protection is not needed during totality. Then a brilliant diamond ring phenomenon appears, seemingly brighter than the same event only minutes ago at second contact. The column of darkness passes off to the east taking its show to others on its path. The whole process then reverses as the moon's edge disappears from the disc of the sun.

A total solar eclipse is a true wonder since the diameter of the sun is 400 times larger than the moon, but since the moon is 400 times closer to earth than the sun the fit is just right- - some of the time.

A total solar eclipse occurs approximately every 18 months, although we have a minimum of two solar eclipses each year. The earth may experience as many as five solar eclipses in a single year although none of those will be total. The moon's rotation around the earth is tilted at approximately a $5^{\circ}$ angle to the equator, and the lunar rotation requires approximately 28 days, so the earth has an eclipse only when the moon passes though the equatorial node in direct line with the sun. This occurs two or three times a year, but during the time of the moon's passage through this node, there can actually be two new moons in one or two of these nodal passes allowing for a total of five possible eclipses. Understanding these cycles, though, is only part of the mystery. The celestial mechanics are imperfect enough that prediction requires knowledge of the zodiac to better understand where the eclipse will actually occur on earth. The "Saros cycle," which determines when and where a particular family of eclipses will occur, is complex and worth a chapter or a book rather than a few paragraphs. The distance of the sun and the moon from the earth are the primary determinants of the duration of the eclipse.

Eclipses have been important to our understanding of the sun's energy as well as that of gravitational lenses. Total solar eclipses in 1919 and 1922 helped prove that gravitational lenses actually bent light and proved Einstein's theory of relativity.

Eclipses are the source of myth including many peculiar beliefs. The Book of Revelation in the Bible speaks of tragedy "... when the sun turns to ashes." Some cultures believe that the unborn child will be harmed in the womb or lost in a miscarriage if its mother views a total eclipse. In other cultures, a pregnant woman may be chided to hold a pot of lime on her abdomen or to lie prone with arms outstretched to prevent birth defects.

The cover photograph was taken during the last total solar eclipse of the millennium as viewed from Harput, Turkey ("Last of the millennium" is true no matter how you view the 2000-1 "beginning of the new millennium" controversy since there was no total solar eclipse in 2000). The diamond ring flashed brilliantly with a few so called "Baily's beads" visible on the leading edge of the moon. "Baily's beads" are those few bright spots of sun visible though the valleys of the moon at the last moment before totality. The slender vibrant ring of red around the sun, the chromosphere, is but a few hundred miles thick and surprisingly, is only $10000^{\circ} \mathrm{F}$, contrasted with the $1000000^{\circ} \mathrm{F}$ or more of the corona. The red prominences of the chromosphere arc thousands of miles free of the surface as if red hot molten lava has been thrown from a volcano. In this photograph, the chromosphere surrounds the edge of the moon and one free prominence can be seen floating off the superior edge of the eclipse, on the right.

This month, June 2001, is in the eclipse season and will witness the first total solar eclipse of the new millennium over southern Africa. The shadow of totality will strike land in Angola and rapidly traverse to the south east, eventually crossing Madagascar, before being extinguished over the southern Indian Ocean.

If you plan to attend, remember to use proper eye protection. Eclipse gazing is a common source of solar retinopathy. Certainly, early observers did not understand solar retinopathy, and it is likely that such pioneers as Galileo developed solar retinopathy. He probably invented the telescope, and was an early champion of solar observation. You may remember what happens when the sun's rays are concentrated with a magnifying lens.

In Galileo's Daughter by Dava Sobel, Galileo is quoted as writing, "As a result of a certain affliction I began to see a luminous halo more than two feet in diameter around the flame of a candle capable of concealing from me all objects, which lay behind it. As my malady diminished, so did the size and density of this halo, though more of it has remained with me than is seen by perfect eyes."

Although Sobel ascribes this to ocular infections transmitted by sharing an eyepiece, it is more likely to be solar retinopathy since Galileo was an avid observer, recording much about the movement of sunspots. I suspect that he did not use a proper solar filter.

If you visit southern Africa for the first total solar eclipse of the third millennium on 21 June 2001, be prepared for the show of a lifetime, and remember that you may find other ophthalmologists beneath centreline.-Ivan R Schwab, MD, UC Davis Department of Ophthalmology, 4860 Y Street, Suite 2400, Sacramento, CA 95817, USA(irschwab@ucdavis.edu). 